\title{
Cerebral damage in diving: Taking the cue from sports concussion medicine
}

\author{
Ann B Shuttleworth-Edwards (PhD) \\ Victoria J Whitefield-Alexander (PhD)
}

National Sports Concussion Initiative, Department of Psychology, Rhodes University, Grahamstown, South Africa

Correspondence to: Ann Edwards (a.edwards@ru.ac.za)

\begin{abstract}
Within the compressed gas diving arena there is a risk of cerebral damage with deleterious neuropsychological sequelae in association with decompression illness (DCI), hypoxia, gas toxicity, as well as the cumulative subclinical effect of 'silent' paradoxical gas embolisms, the last being an area of growing concern. However, within diving medicine there is little evidence of the regular use of neuropsychological evaluation to monitor brain-related sequelae of frequent diving activity. In contrast, in recent years there has been an explosion of interest in the management of sports concussive injury, including emphasis on the pivotal role of neuropsychological evaluation within that context. Taking the cue from sports concussion medicine, it is proposed that there is an urgent need to incorporate neurocognitive baseline and follow-up screening as a core component in the medical management of those involved in intensive commercial and recreational compressed air diving activities. The objective would be to facilitate (i) accurate neurodiagnostic follow-up of frank DCI or an identifiable hypoxic or toxic incident; (ii) timeous identification of cumulative deleterious effects of repetitive subclinical hypoxic/toxic incidents and/or 'silent' paradoxical gas embolisms that might affect them in later life; and (iii) disability assessment following any such events or the combination thereof for rehabilitation and compensation purposes.
\end{abstract}

\section{Brain damage risk in diving}

There is a substantial body of research on the neurological and neuropsychological effects of compressed gas diving that occurs in both the recreational and occupational spheres. A recent review of diving practice indicates that there are approximately 7 million divers active worldwide, and about 500000 training every year, ${ }^{1}$ despite the fact that it is accompanied by a number of brain-related hazards including hypoxia, gas toxicity, bubble-related insults and decompression illness (DCI).* In a survey of 770 divers Beckett and Kordick ${ }^{2}$ indicated that half of these divers (53\%) had suspected decompression symptoms. Similarly, in a study of 156 air and saturation divers $51 \%$ had DCI,

${ }^{*}$ The reader is referred elsewhere for a more comprehensive delineation of the physiological mechanisms involved in injury to the nervous system in association with diving, including decompression illness, hypoxia, gas toxicity and 'silent' paradoxical gas embolisms, ${ }^{4,525}$ which are beyond the scope of the current article.
$33 \%$ of these with neurological symptoms. In contrast to the high risk of DCI implied by these figures, Vann et al. ${ }^{4}$ suggest that if appropriate decompression procedures are followed, DCI is uncommon and incidence declines. They suggest that acceptable risk for commercial diving is $0.1 \%$ for mild cases and $0.025 \%$ for serious cases; for US Navy diving acceptable risk is $2 \%$ for mild cases and $0.1 \%$ for serious cases. However, these authors concede that there are insufficient data for accurate estimates of incidence of decompression sickness both in the recreational and commercial spheres, and that incidence figures based on dives made well within maximum exposure limits of accepted procedures are an underestimate of the true rates at maximum limits. Notwithstanding uncertainty around the rate of occurrence of frank DCI, there is growing emphasis on the fact that divers may be suffering subtle symptoms that they do not recognise as DCI, and/or be suffering subclinical effects of repetitive 'silent' paradoxical gas embolisms that may have long-term deleterious neurological consequences. ${ }^{5,6}$

As reviewed in Wilmhurst, ${ }^{6}$ neuroradiological investigations on divers predominantly provide support for brain-related changes in association with diving. A series of studies have investigated long-term/cumulative neurological and neurocognitive effects via objective psychometric testing of divers in contrast to demographically matched non-diving controls, with outcome implicating deleterious CNS effects. Outcomes included prolonged reaction time scores for construction divers; significantly poorer learning and short-term memory performance, and a tendency to sacrifice accuracy for speed for commercial abalone divers; ${ }^{8}$ and performance deficits in number of reference memory errors and navigation behaviour of construction divers. 'A cohort of divers who complained of forgetfulness were differentiated from divers without this complaint, on the basis of being significantly more impaired with regard to objective memory tests.

Further of note are studies that provide evidence for cumulative deleterious effects in association with repeat-diving. The study of Aarli et al.," investigating for CNS dysfunction, documented no significant changes in any of the professional divers immediately after one dive, but there was clear-cut impairment in a group who had performed two dives three months apart. Research by Vaernes et al. ${ }^{12}$ points to how repeated deep-diving activity is likely to result in greater neuropsychological impairment. It appears that, for professional divers with high exposure to decompression stress, the incidence of intellectual decline may be as high as $50 \%$, and accordingly the concern is expressed that diving may produce residual intellectual decline equivalent to dementia or 'punch-drunk syndrome' as described in association with years of competitive participation in boxing. Finally, a very recent study of military divers without a history of DCI demonstrated higher motor and decision reaction times among very experienced divers logging extensive diving hours, 
compared with less experienced military divers with significantly fewer diving hours. This study points to the possibility that cerebral lesions may occur after diving even without apparent DCI.

In essence, the neurocognitive decrements reported across the above-cited studies in association with diving participation fall broadly within the ambits of short-term memory, attentional abilities, processing speed and reaction time.

\section{Parallels between cerebral injury in field contact sport and diving}

Despite very different neuropathological underpinnings, it is apparent that from a neuropsychological perspective a number of parallels can be drawn between cerebral injury sustained via participation in a field contact sport and compressed gas diving activity. Reviews indicate that the incidence of sustaining a concussion in American football or Rugby Union at adult level over a series of seasons is $42 \%$ and $50 \%$, respectively. ${ }^{14}$ Declines in neurocognitive function are more clearly evident when there is a history of two or more concussions and more extended periods of high-level participation in the sport, and it is commonly acknowledged that effects may go unrecognised or be subclinical, yet have cumulative deleterious longterm effects. ${ }^{15}$ Finally, there is a substantial body of research that points to permanent neurocognitive consequences of concussive and cumulative subconcussive injury in association with American football, soccer, and Rugby Union, ${ }^{15}$ including diminished short-term memory, processing speed, reaction time, and attentional abilities.

Accordingly, while the incidence of concussion documented in the rugby-football sports appears considerably higher than the apparent incidence of DCI under suitably controlled conditions, ${ }^{4}$ the following broad-based similarities exist between the two contexts. Firstly, to a greater or lesser extent, there is known risk for the frank occurrence of a cerebrally noxious event to take place (i.e. concussion or DCI) that may/may not be accompanied by persisting neuropsychological dysfunction. In both forums, albeit for different reasons, there is growing concern for 'silent' subclinical neurological damage in association with participation in these activities, with deleterious neurocognitive consequences. Evidence for the presence of such neuropsychological dysfunction is enhanced in association with prolonged, repetitive and/or professional level participation in each of these activities. Finally, the neurocognitive consequences in association with cerebral damage sustained through participation in both contact sport and diving, albeit of different neuropathological origin, are nevertheless functionally similar, falling broadly within the areas of short-term memory, attentional abilities, processing speed and reaction time. Therefore, from a neuropsychological perspective, equivalent screening mechanisms for deleterious outcome due to cerebral damage that may occur in association with contact sport and diving (although neuropathogically distinct) are entirely in order.

\section{Routine screening for neuropsychological impairment in divers}

Despite the parallels that can be drawn around the occurrence and long-term outcome of neurological damage in association with concussion and diving-related brain impairment, there are marked differences in terms of how this aspect is medically managed within the two arenas. With regard to sports concussion, there has been an explosion of interest in the problem over the last decade, including the convening of three international conferences to initiate up-todate recommendations for optimal medical management of the concussive injury. ${ }^{16-18}$ One of the core guidelines to emerge from these seminal meetings is that individualised medical management of the concussed athlete is recommended including the use of neuropsychological assessment, optimally under the guidance of neuropsychologists who are considered to be 'in the best position' to interpret neuropsychological tests. ${ }^{18,19}$

In contrast, underwater medicine appears to be a relatively neglected area, notably in terms of neuropsychological involvement, with the main attention still being paid to emergency care of respiratory crises with neurological consequences, rather than long-term aftercare including the assessment of disability for compensation purposes. ${ }^{20.21}$ Ironically, there was an international consensus conference on the long-term health effects of diving that pre-dated the first of the concussion in sport consensus conferences ${ }^{22}$ at which longitudinal follow-up of divers was recommended. However, it appears that the convening of a forum of this type out of concern for the medical management of divers in particular (rather than hyperbaric medicine more generally) was not repeated, nor did it result in the implementation of neurocognitive screening on a routine or wide basis. A comprehensive Pubmed and Biomed search confirms the impression that any formally stated recommendation concerning neuropsychological evaluation as a routine part of the medical examination of commercial or recreational divers has never been in place, and continues to be neglected.

Specific issues pertaining to neuropsychological assessment A number of neurocognitive screening programmes based on well-researched traditional neuropsychological test stimuli (i.e. having good face validity as neuropsychological instruments) have been especially developed within the sports concussion arena. These might be considered for use in the diving context, e.g. ANAM, Headminder and ImPACT ${ }^{23}$ Of these the ImPACT programme, now in its fourth updated version, is the most commonly employed test of its kind worldwide, and is an HPCSAapproved test for use within South Africa. The recommended mechanism within the sports arena is to conduct baseline neurocognitive evaluation on individuals prior to engagement in the cerebrally hazardous activity using a recognised instrument of this type for comparative purposes with follow-up testing after a cerebrally harmful event.

Specifically, the ImPACT test provides an automated report on six cognitive composites that incorporate the functional areas described above that are typically implicated following the decompression event, viz. memory function, attentional abilities, reaction time and processing speed. A strength of the ImPACT programme is the incorporation of a 24 -item check list of neuropsychiatric symptoms that are typically in evidence after concussion. Recently, a special adaptation of ImPACT has been developed for work within the military, keeping the neurocognitive aspect intact, but making adjustments to the demographic and symptom questionnaires. ${ }^{24}$ Similarly, for use within the diving context it would be possible to tailor-make the programme to include relevant details such as prior history of any incident of DCI, years involved in diving, estimate of number of dives completed, and purpose of diving. Furthermore, it would be possible to ensure that the symptom checklist incorporates neuropsychiatric sequelae that have regularly been described in association of decompression illness. Symptoms following diffuse cerebral presentations such as concussion and 
DCI vary widely and are nonspecific, with many sequelae in common, such as 'dizziness', 'headache', 'difficulty remembering', 'numbness', 'tingling', 'difficulty concentrating' and 'fatigue'. From a neuropsychological point of view, it is of prime diagnostic value to have a testee's idiosyncratic self-report symptom profile, in addition to the objective psychometric test profile, when drawing conclusions concerning the presence or absence of neurological fall-out and/or the extent of disability.

\section{Conclusions}

As a matter of some urgency, it would seem appropriate for diving medicine to consider taking the cue from sports concussion medicine, specifically with regard to the incorporation of neuropsychological evaluation (such as is widely applied within professional and amateur contact sports) to monitor its cerebrally hazardous injury. Ideally, this would involve the initiation of computerised neurocognitive baseline screening, and follow-up testing in the wake of any DCI incident, as well as other identifiable hypoxic or toxic respiratory incidents, to establish the extent of disability. Importantly, comparative baseline screening for divers on an annual or a bi-annual basis would provide the platform to establish the presence of 'silent' cognitive decline implicating the insidious onset of brain dysfunction that may affect them in the long term, such as may not be immediately in the diver's awareness or apparent during a regular medical examination.

As DCI appears to be a relatively uncommon and declining occurrence, especially when under controlled conditions (compared with the incidence of the concussive incident in contact sport), the initiation of routine neurocognitive baseline testing in all contexts might be deemed to be unwarranted. Nevertheless, it is still a distinct risk, and taken together with the growing concern for subclinical neuropathological changes due to paradoxical gas embolisms, such testing would appear to be the advised route to follow for those involved in recurrent compressed gas diving activity. At the very least, the implementation of longitudinal studies of both occupational and recreational divers utilising computerised neurocognitive testing with a tool such as ImPACT is indicated, to capture immediate and enduring neuropsychological outcome of both clearly identifiable as well as 'silent' neuropathological events.

\section{Acknowledgements}

This article is based on a paper presented by invitation at the 17th International Congress of Hyperbaric Medicine (ICHM), Cape Town Convention Centre, Cape Town, South Africa, 16 - 19 March 2011.

Funding for costs involved in the preparation and earlier presentation of this review was facilitated by National Research Fund (NRF) incentive funding, and a Rhodes University Joint Research Council (JRC) grant.

\section{Declaration of conflict of interests}

The authors are involved in implementing the ImPACT programme in South Africa and the UK for research and clinical purposes.

\section{REFERENCES}

1. Levett D, Millar I. Bubble trouble: a review of diving physiology and disease. Postgrad Med J 2008;84(997):571-578.

2. Beckett A, Kordick M. Risk factors for dive injury: a survey study. Research in Sports Medicine 2007;(15):3-201.

3. Todnem K, Nyland H, Kambestad B, Aarli J. Influence of occupational diving upon the nervous system: an epidemiological study. British Journal of Industrial Medicine 1990;47(708):714.

4. Vann R, Butler F, Mitchell S, Moon R. Decompression illness. Lancet 2011;377(9760):153-164.

5. Kowalski J, Varn A, Rottger S, et al. Neuropsychological deficits in scuba divers: an exploratory investigation. Undersea Hyperbaric Medicine 2011;38(3):197-204.

6. Wilmhurst P. Brain damage in divers. BMJ 1997;314(7082):689-690.

7. Bast-Pettersen R. Long-term neuropsychological effects in non-saturation construction divers. Aviation Space and Environmental Medicine 1999;70(1):51-57.

8. Williamson A, Clarke B, Edmonds C. The influence of diving variables on perceptual and cognitive functions in professional shallow-water (abalone) divers. Environ Res 1989;50(1):93-102.

9. Leplow B, Tetzlaff K, Holl D, Zeng L, Reuter M. Spatial orientation in contruction divers - are there associations with diving experience? Int Arch Occup Environ Health 2001;74(3):189-198.

10. Taylor C, Macdiarmid J, Ross J, et al. Objective neuropsychological test performance of professional divers reporting a subjective complaint of "forgetfulness or loss of concentration". Scand J Work, Environ Health 2006;32(4):310-317.

11. Aarli J, Vaernes R, Brubakk A, Nyland H, Skeidsvoll H, Tonjum S. Central nervous dysfunction associated with deep-sea diving. Acta Neurol Scand 1985;71(1):2-10.

12. Vaernes R, Klove H, Ellertsen B. Neuropsychologic effects of saturation diving. Undersea Biomedical Research 1989;16(3):233-251.

13. Edmonds C, Boughton J. Intellectual deterioration with excessive diving (punch drunk divers). Undersea Biomedical Research 1985;12(3):321-326.

14. Shuttleworth-Edwards AB, Noakes TD, Radloff SE, et al. The comparative incidence of reported concussions for follow-up management in South African Rugby Union. Clin J Sport Med 2008;18(5):403-409.

15. Shuttleworth-Edwards AB, Whitefield VJ. Ethically we can no longer sit on the fence: A neuropsychological perspective on the cerebrally hazardous contact sports. South African Journal of Sports Medicine 2007;19(2):32-38.

16. Aubry M, Cantu R, Dvorak J, et al. Summary and agreement statement of the 1st International Symposium on Concussion in Sport, Vienna, 2001. Clin J Sport Med 2002;12(1):6-11.

17. McCrory P, Johnston K, Meeuwisse W, et al. Summary and agreement statement of the 2nd International Conference on Concussion in Sport, Prague, 2004. Clin J Sport Med 2005;15(2):48-55.

18. McCrory P, Meeuwisse W, Johnston K, et al. Consensus statement on concussion in sport: the 3rd International Conference on Concussion in Sport, Zurich, November 2008. Br J Sports Med 2009;43:i76-i84.

19. Schatz P, Moser RS. Current issues in pediatric sports concussion. The Clinical Neuropsychologist 2011;25(6):1042-1057.

20. Braatvedt G, Mathew BG, Corrall R. Underwater medicine: a neglected area in accident and emergency specialist training. Br J Sports Med 1991;25(2):102-103.

21. Rozali A, Khairuddin H, Sherina M, Zin B, Sulaiman A. Decompression illness secondary to occupational diving: recommended management based current legislation and practice in Malaysia. Med J Malaysia 2008;63(2):166-169.

22. Hope A, Lund T, Elliot D, Halsey M, Wiig H. Long term health effects of diving. An International Consensus Conference, Godoysund, Norway, 6 - 10 June 1993. Bergen: Norwegian Underwater Technology Centre/University of Bergen, 1994.

23. Guskiewicz KM, Bruce DE, Cantu RC, et al. National Athletic Trainers' Association position statement: Management of sport-related concussion. Journal of Athletic Training 2004;39:280-297.

24. Lovell MR, Collins M, Pardini JE, Parodi A, Yates A. Management of cerebral concussion in military personnel: lessons learned from sports medicine. Operative Techniques in Sports Medicine 2005;13:212-221.

25. Lishman W. Organic Psychiatry: The Psychological Consequences of Cerebral Disorder. 3rd ed. Oxford, UK: Blackwell Science Ltd., 1999. 